\title{
MICROALBUMINURIA AS A PREDICTOR OF PREGNANCY-INDUCED HYPERTENSION
}

\author{
V. Kasthuri', S. Thangathai ${ }^{2}$
}

${ }^{1}$ Associate Professor, Department of Obstetrics and Gynaecology, Government Thiruvarur Medical College. ${ }^{2}$ Associate Professor, Department of Obstetrics and Gynaecology, Government Thiruvarur Medical College.

ABSTRACT

\section{BACKGROUND}

Proteinuria is considered to be the central feature in the diagnosis of pre-eclampsia and the amount of proteinuria has shown to correlate with increasing maternal and perinatal morbidity and mortality.

\section{AIM OF THE STUDY}

The aim of the present prospective study was to know the predictive value of microalbuminuria in predicting the development of Pregnancy-induced hypertension, Pre-eclampsia, Transient hypertension.

\section{OBJECTIVES}

a) Determining urinary microalbumin concentration with increasing gestation in women who remain normal.

b) To correlate microalbuminuria with various indices like age, parity, weight, gestational age, and platelet count.

\section{STUDY DESIGN}

This is a prospective cohort study.

\section{MATERIALS AND METHODS}

The present prospective study was done in 200 pregnant women at 18-22 weeks of gestation to know the predictive value of microalbuminuria in predicting possibilities of development of PIH/Pre-eclampsia/Transient hypertension. The concentration of urine microalbumin was estimated by the Indirect Latex Slide Test.

\section{RESULTS}

Microalbuminuria seems to be a clinical test, which can be used for the prediction of PIH, pre-eclampsia around 18-22 weeks, later on it is not useful. Microalbuminuria is a useful predictor in primigravida and obese women.

\section{CONCLUSION}

Urine albumin excretion increases as the disease severity increases. It may be concluded that early pregnancy levels of microalbuminuria can be used as predictor of pre-eclampsia with high negative predictive value.

\section{KEYWORDS}

Microalbuminuria, Pre-eclampsia, Latex Slide Test.

HOW TO CITE THIS ARTICLE: Kasthuri V, Thangathai S. Microalbuminuria as a predictor of pregnancy-induced hypertension. J. Evolution Med. Dent. Sci. 2016;5(68):4868-4875, DOI: 10.14260/jemds/2016/1107

\section{INTRODUCTION}

Hypertensive disorders complicating pregnancy are common and form one of the deadly triad along with haemorrhage and infection that results in much of the maternal morbidity related to pregnancy. It affects $4 \%$ to $5 \%$ of all pregnancies. Hypertensive disorders continue to be rampant globally as aetiology not well known. The risk factors are nonspecific and so there is lack of sensitive predictive test.(1) Several methods of identifying pregnant women who are at risk for preeclampsia have been proposed. These tests, however, have had limitations as screening tools in the clinical setting because of their complexity, the high incidence of false-positive results, or the subjective nature of result interpretation. Proteinuria

Financial or Other, Competing Interest: None.

Submission 19-07-2016, Peer Review 12-08-2016,

Acceptance 17-08-2016, Published 23-08-2016.

Corresponding Author:

Dr. V. Kasthuri,

\#13/26, 1st Main Road,

Kalaimagal Nagar, Ekkaduthangal,

Chennai-600032.

E-mail: sdr.kasthuri@yahoo.co.in

DOI: $10.14260 /$ jemds/2016/1107 has classically been an important sign in the diagnosis of preeclampsia.(2,3) However, customary dipstick methods for detecting proteinuria fail to detect minimal elevations in urinary excretion of albumin that maybe present before other clinical signs and symptoms of pre-eclampsia. With the recent development of the Indirect Latex Slide Test for detection of microalbuminuria, it is now possible to detect minimal elevations in albumin excretion that would have gone unnoticed in the past. Microalbuminuria is defined as excretion of minute amount of urinary albumin concentration between $30-300 \mathrm{mg} /$ litre and urinary albumin excretion rate of $20-200 \mu \mathrm{g} / \mathrm{min}$, which is usually not detectable by routine screening methods. $(4,5)$ Till it manifests overtly as proteinuriaa condition where significant kidney damage has already occurred.

\section{MATERIALS AND METHODS}

The study group consisted of 200 pregnant women at 18-22 weeks of gestation, free of signs and symptoms of preeclampsia, and pregnancy-induced hypertension. They were registered into the study at random from Outpatient Clinic of 
Obstetrics Department of Government Kasturba Gandhi Hospital, Chennai.

\section{Inclusion Criteria}

200 pregnant women at 18-22 weeks of gestation, free of signs and symptoms of pre-eclampsia, and pregnancy-induced hypertension.

\section{Exclusion Criteria}

a) Past history of chronic hypertension, diabetes, renal disease.

b) Presence of urinary tract infection.

c) Presence of genital tract infection.

d) $\mathrm{BP} \geq 140 / 90 \mathrm{mmHg}$ at the time of gestation.

e) Presence of proteinuria by customary dipstick method.

\section{All these Patients had Completed their Initial Evaluation}

\section{like}

a) Complete history.

b) General examination-BP was measured after 5 minutes in the supine position and oedema evaluated clinically.

c) Per abdominal examination.

d) Routine blood investigations.

e) Urine examination-routine and microalbuminuria.

\section{METHODS}

At each clinical visit, a random clean catch sample of urine was collected in a sterile glass bottle without any preservative and analysed for microalbuminuria by indirect latex slide test. Standardisation of microalbumin estimation was done in 50 nonpregnant normotensive women of similar age and parity. Microalbumin excretion was expressed as mg/ltr. and was taken positive when microalbumin excretion was $25 \mathrm{mg} / \mathrm{ltr}$. The concentration of urine microalbumin was established by the indirect latex slide test.

\section{Reagent}

1. Anti-human albumin reagent: The concentration of antibodies to human albumin is adjusted to provide sensitivity of about $>=25 \mathrm{mg} /$ litre and above of microalbuminuria.

2. Albumin latex reagent: A uniform suspension of polystyrene latex particles to which human albumin has been chemically coupled.

\section{Principle of Indirect Latex Slide Test}

Microtex slide test for the detection of microalbuminuria is based on the principle of agglutination inhibition. The urine specimen to be tested is first mixed with antibody reagent containing antibodies directed against human albumin. The latex coupled with human albumin is added to the mixture and is allowed to react. When the urine specimen does not contain albumin, antibodies to human albumin would be free to react with latex coupled with human albumin causing agglutination. When the urine specimen contains at least $25 \mathrm{mg} / \mathrm{litre}$ of albumin, the antibodies to human albumin will be neutralised and not available to react with latex coupled with human albumin. Hence, no agglutination will be observed.

\section{Sample Collection and Preparation}

Specimen collected in clean glass or plastic containers free of detergents. Specimen tested preferably within $12 \mathrm{hrs}$. of collection.
If delay in testing occurs thiomersal $(0.01 \%)$ added to the specimen and stored at $2-8^{\circ} \mathrm{C}$ up to 72 hours.

\section{Test Procedure}

1. All reagents and samples are brought to room temperature before use.

2. One drop of clear urine under test is placed vertically on the glass slide.

3. One drop of antihuman albumin reagent is added to the drop of urine under test.

4. The antihuman albumin and reagent is mixed over the circle for 30 seconds.

5. One drop of well mixed albumin latex reagent is added to the mixture uniformly over the entire circle.

6. The slide is rocked gently back and forth observing for agglutination macroscopically at 3 minutes.

\section{Interpretation of Results}

Agglutination-negative test indicating absence of detectable levels of albumin in urine signifying absence of microalbumin. No agglutination-positive test indicating presence of albumin in concentration of above $25 \mathrm{mg} /$ litre in urine signifying microalbuminuria.

The urinary microalbumin estimation was done minimum 3 times during pregnancy at 18-22 wks., 23-28 wks., and 29$34 \mathrm{wks}$. All subjects received antenatal care and were followed for occurrence of hypertensive disorder.

\section{RESULTS}

Of the 200 subjects, 186 could be followed up. Out of 186 subjects who went to term and in which outcome could be known, 41 (22.35\%) developed hypertensive disorder (PIH, Pre-eclampsia, Transient hypertension). Of these 41 women, $23(12.30 \%)$ had $\mathrm{PIH}, 10(5.20 \%)$ pre-eclampsia, and 8 (4.71\%) developed transient hypertension. At 18-22 weeks, $4.96 \%$ were microalbuminuria positive $66 \%$ of them and out of microalbuminuria negative $20 \%$ developed hypertensive disorder. By 29-34 weeks of gestation, $96.76 \%$ were microalbuminuria positive, $23.75 \%$ of them and $18 \%$ of microalbumin negative also developed hypertensive disorder by term. Analysis of variance of microalbuminuria positivity with socioeconomic status, height, past gestational complications, $\mathrm{Hb}$, and blood group was found to be nonsignificant, but with weight was significant. Gravidity was found to be a statistically significant variable. Microalbuminuria estimation around 18 weeks seems to be a useful predictor especially in heavier women and primigravidas. $(6,7,8)$ The sensitivity of microalbuminuria in predicting the development of PE was $60 \%$, specificity $96.9 \%$, Positive Predictive Value (PPV) 66.6\%, and Negative Predictive Value (NPV) $80 \%$. It may be concluded that early pregnancy levels of microalbuminuria can be used as predictors of pre-eclampsia with high negative predictive value. $(9,10)$

\section{Out of 200 Subjects included at 18-22 Weeks}

- 186 women could be investigated by 23-28 weeks.

- 2 women aborted

- 12 women were lost to follow up.

- 186 samples could be analysed serially by 29-34 weeks. These 186 subjects were further followed. 


\section{Out of the 186 Subjects}

1.145 women $(77.65 \%)$ remained normotensive.

2. 41 women (22.35\%) developed hypertensive disorder.

\section{Pattern of HT Disorders in Microalbuminuria Tested} Patients

Of the 41 women (22.35\%) developed hypertensive disorder.

a. 23 women (12.3\%) developed Pregnancy-Induced Hypertension (PIH).

b. 10 women (5.2\%) developed Pre-eclampsia.

c. 8 women (4.7\%) developed Transient hypertension.

Out of the $33(17.7 \%)$ Women with (PIH)/Pre-eclampsia

a. 23 women (12.5\%) had Pregnancy-Induced Hypertension (PIH).

b. 7 women (4.1\%) had mild Pre-eclampsia.

c. 3 women (1.1\%) had severe Pre-eclampsia.

\section{Observations in Microalbuminuria Tested}

Patients at 18-22 Weeks of Gestation. At 18-22 Weeks:

1. 9 women (4.96\%) were microalbumin positive. Of these;

a. 3 women (33\%) remained normotensive.

b. 6 women (66\%) developed hypertensive disorder.

2. 177 women (95.04\%) were microalbuminuria negative. Of these;

a. 142 women $(80 \%)$ remained normotensive.

b. 35 women $(20 \%)$ developed hypertensive disorder.

\section{Observations in Microalbuminuria Tested}

Patients at 23-28 Weeks of Gestation. By 23-28 weeks:

1. 65 women $(34.92 \%)$ were microalbumin positive. Of these;

a. 40 women $(62 \%)$ remained normotensive.

b. 25 women (38\%) developed hypertensive disorder.

2. 121 women $(65.18 \%)$ were microalbuminuria negative. Of these;

a. 107 women $(88.2 \%)$ remained normotensive.

b. 14 women (11.8\%) developed hypertensive disorder.

\section{Observations in Microalbuminuria Tested}

Patients at 29-34 Weeks of Gestation. By 29-34 Weeks:

1. 180 women $(96.76 \%)$ were microalbumin positive. Of these;

a. 137 women $(76.25 \%)$ remained normotensive.

b. 43 women (23.75\%) developed hypertensive disorder.

2. 6 women $(3.24 \%)$ were microalbuminuria negative. Of these;

a. 5 women (82\%) remained normotensive.

b. 1 woman $(18 \%)$ developed hypertensive disorder.

Observation in Microalbuminuria Tested Patients at Various Weeks of Gestation in Relation to Age

Analysis of variance for microalbuminuria positivity and age was found to be significant. ( $\mathrm{p}<0.001)$. The microalbuminuria and hypertensive disorders was more in the extremes of age (18-19 yrs. and 28-33 yrs.) at 18-22 weeks of gestational age.

Observations in Microalbuminuria Tested Patients at 1822 Weeks of Gestation in Relation to Age at 18-22 Weeks In the age group of 18-22 years-Out of 65 women:

1. 3 women were microalbumin positive. Of these;

a. Women remained normotensive.

b. Women developed hypertensive disorder.
2. 62 women were microalbuminuria negative. Of these;

a. 59 women remained normotensive.

b. 3 women developed hypertensive disorder.

In the age group of 23-27 years-Out of 80 women:

1. 1 woman were microalbumin positive and developed hypertensive disorder.

2. 79 women were microalbuminuria negative. Of these;

a. 77 women remained normotensive.

b. 2 women developed hypertensive disorder.

In the age group of 28-33 years-Out of 41 women.

1. 5 women were microalbumin positive. Of these;

a. 2 women remained normotensive.

b. 3 women developed hypertensive disorder.

2. 36 women were microalbuminuria negative. Of these;

a. 6 women remained normotensive.

b. 30 women developed hypertensive disorder.

Observations in Microalbuminuria Tested Patients at Various Weeks of Gestation in Relation to Gravidity

Gravidity was found to be statistically significant variable. More microalbuminuric primigravida developed hypertensive disorder. $(\mathrm{p}<0.001)$.

Observations in Microalbuminuria Tested Patients at 1822 Weeks of Gestation in Relation to Gravidity at 18-22 Weeks

In gravida 1-Out of 94 women:

1. 7 women were microalbumin positive. Of these;

a. 3 women remained normotensive.

b. 4 women developed hypertensive disorder.

2. 87 women were microalbuminuria negative. Of these;

a. 71 women remained normotensive.

b. 16 women developed hypertensive disorder.

In gravida 2-Out of 65 women:

1. None of the women were microalbumin positive.

2. 65 women were microalbuminuria negative. Of these;

a. 56 women remained normotensive.

b. 9 women developed hypertensive disorder.

In gravida 3 and above-Out of 27 women:

1. 2 women were microalbumin positive and all of them developed hypertensive disorder.

2. 25 women were microalbuminuria negative. Of these;

a. 15 women remained normotensive.

b. 10 women developed hypertensive disorder.

Observations in Microalbuminuria Tested Patients at Various Weeks of Gestation in Relation to Maternal Weight

Analysis showed maternal weight as an independent variable with microalbuminuria for predicting hypertensive disorder at 18-22weeks.

Observations in Microalbuminuria Tested Patients at 1822 Weeks of Gestation in Relation to Maternal Weight at 18-22 Weeks

In women weighing $45-49 \mathrm{~kg}$-Out of 19 women:

1. 2 women were microalbumin positive. Of these;

a. 1 women remained normotensive.

b. 1 women developed hypertensive disorder. 
2. 17 women were microalbuminuria negative. Of these;

a. 15 women remained normotensive.

b. 2 women developed hypertensive disorder.

In women weighing 50-54 Kg-Out of 135 women:

1. 7 women were microalbumin positive. Of these;

a. 2 women remained normotensive.

b. 5 women developed hypertensive disorder.

2. 128 women were microalbuminuria negative. Of these;

a. 102 women remained normotensive.

b. 26 women developed hypertensive disorder.

In women weighing $>55 \mathrm{Kg}$-Out of 32 women.

All women were microalbuminuria negative. Of these;

a. 25 women remained normotensive.

b. 7 women developed hypertensive disorder.

Observations in Microalbuminuria Tested Patients at Various Weeks of Gestation in Relation to Platelet Count Platelet count $<1.5$ lakhs/cubic. $\mathrm{mm}$ was found to be correlated to microalbuminuria significantly at 18-22 weeks $(\mathrm{P}<0.001>)$.

Observations in Microalbuminuria Tested Patients at 1822 Weeks of Gestation in Relation to Platelet Count at 1822 Weeks.

In women with platelet count $\leq 1.5$ lakhs/cubic. mm-Out of 22 women:

\begin{tabular}{|c|c|c|c|c|c|}
\hline Author of Study & No. of Subjects & Sensitivity & Specificity & $\begin{array}{c}\text { Positive } \\
\text { Predictive Value }\end{array}$ & $\begin{array}{c}\text { Negative } \\
\text { Predictive Value }\end{array}$ \\
\hline 1. Irgine et al & 54 & $61 \%$ & $87 \%$ & $61 \%$ & $87 \%$ \\
\hline 2. Rodriquez & 106 & $50 \%$ & $82 \%$ & $26 \%$ & $93 \%$ \\
\hline 3. Das et al & 98 & $68.4 \%$ & $91.8 \%$ & $56 \%$ & $94 \%$ \\
\hline 4. Moller & 124 & $61 \%$ & $87 \%$ & $61 \%$ & $87 \%$ \\
\hline 5. Lopez-Spinoza et al & 88 & $52 \%$ & $82 \%$ & $26 \%$ & $93 \%$ \\
\hline 6. Our Study & 200 & $60 \%$ & $96.9 \%$ & $66.6 \%$ & $80.2 \%$ \\
\hline \multicolumn{7}{l}{} \\
\hline
\end{tabular}

\begin{tabular}{|c|c|c|c|c|c|}
\hline \multirow{2}{*}{ No. of Patients with Hypertensive Disorders } & \multicolumn{2}{|c|}{ PIH } & \multicolumn{2}{c|}{ Pre-eclampsia } & \multirow{2}{*}{ Transient Hypertension } \\
\cline { 2 - 5 } & Mild & Severe & Mild & Severe & \\
\hline $41(22.35 \%)$ & $21(10.5 \%)$ & $2(1.7 \%)$ & $7(4.1 \%)$ & $3(1.1 \%)$ & $8(4.7 \%)$ \\
\hline \multicolumn{4}{|c|}{ Pattern of HT Disorders in Microalbuminuria Tested Patients } \\
\hline
\end{tabular}

\begin{tabular}{|c|c|c|c|c|c|c|}
\hline $\begin{array}{c}\text { Gestational Age in } \\
\text { Weeks }\end{array}$ & \multicolumn{2}{|c|}{ Microalbuminuria } & \multicolumn{2}{c|}{ Microalbuminuria Positive } & \multicolumn{2}{c|}{ Microalbuminuria Negative } \\
\hline \multirow{2}{*}{$18-22$} & Positive & Negative & Normotensive & Hypertensive & Normotensive & Hypertensive \\
\cline { 2 - 7 } & $9(4.96 \%)$ & $177(95.04 \%)$ & $3(33 \%)$ & $6(66 \%)$ & $142(80 \%)$ & $35(20 \%)$ \\
\hline
\end{tabular}

\begin{tabular}{|c|c|c|c|c|c|c|}
\hline $\begin{array}{c}\text { Gestational Age } \\
\text { in Weeks }\end{array}$ & \multicolumn{2}{|c|}{ Microalbuminuria } & \multicolumn{2}{c|}{ Microalbuminuria Positive } & \multicolumn{2}{c|}{ Microalbuminuria Negative } \\
\hline \multirow{2}{*}{$23-28$} & Positive & Negative & Normotensive & Hypertensive & Normotensive & Hypertensive \\
\cline { 2 - 7 } & $65(34.92 \%)$ & $121(65.18 \%)$ & $40(62 \%)$ & $25(38 \%)$ & $107(88.2 \%)$ & $14(11.8 \%)$ \\
\hline \multicolumn{3}{|c|}{ Observations in Microalbuminuria Tested Patients at 23-28 Weeks of Gestation } \\
\hline
\end{tabular}

\begin{tabular}{|c|c|c|c|c|c|c|}
\hline $\begin{array}{c}\text { Gestational Age } \\
\text { in Weeks }\end{array}$ & \multicolumn{2}{|c|}{ Microalbuminuria } & \multicolumn{2}{c|}{ Microalbuminuria Positive } & \multicolumn{2}{c|}{ Microalbuminuria Negative } \\
\hline \multirow{2}{*}{$29-34$} & Positive & Negative & Normotensive & Hypertensive & Normotensive & Hypertensive \\
\cline { 2 - 7 } & $180(96.76 \%)$ & $6(3.24 \%)$ & $137(76.25 \%)$ & $43(23.75 \%)$ & $5(82 \%)$ & $1(18 \%)$ \\
\hline \multicolumn{3}{|c|}{ Observations in Microalbuminuria Tested Patients at 29-34 Weeks of Gestation } \\
\hline
\end{tabular}




\begin{tabular}{|c|c|c|c|c|c|}
\hline \multirow{2}{*}{$\begin{array}{c}\text { Age of Patient } \\
\text { in Years }\end{array}$} & \multicolumn{2}{|c|}{ Microalbuminuria Positive } & \multicolumn{2}{|c|}{ Microalbuminuria Negative } & \multirow{2}{*}{ Subjects } \\
\hline & Normotensive (Weeks) & Hypertensive (Weeks) & Normotensive (Weeks) & Hypertensive (Weeks) & \\
\hline $18-22$ & $1(33.3 \%)$ & $2(33.3 \%)$ & $59(41.5 \%)$ & $3(8.5 \%)$ & $65(34.9 \%)$ \\
\hline $23-27$ & - & $1(16.6 \%)$ & $77(54.2 \%)$ & $2(5.7 \%)$ & $80(43 \%)$ \\
\hline $28-33$ & $2(66.7 \%)$ & $3(50 \%)$ & $6(4.2 \%)$ & $30(85.7 \%)$ & $41(22 \%)$ \\
\hline Total & 3 & 6 & 142 & 35 & 186 \\
\hline
\end{tabular}

\begin{tabular}{|c|c|c|c|c|c|}
\hline \multirow[b]{2}{*}{ Gravidity } & \multicolumn{2}{|c|}{ Microalbuminuria Positive } & \multicolumn{2}{|c|}{ Microalbuminuria Negative } & \multirow[b]{2}{*}{ Subjects } \\
\hline & $\begin{array}{l}\text { Normotensive } \\
\text { (Weeks) }\end{array}$ & $\begin{array}{l}\text { Hypertensive } \\
\text { (Weeks) }\end{array}$ & Normotensive (Weeks) & $\begin{array}{l}\text { Hypertensive } \\
\text { (Weeks) }\end{array}$ & \\
\hline Gravida-1 & $3(75 \%)$ & $4(60 \%)$ & $71(50 \%)$ & $16(45.7 \%)$ & 97 (53\%) \\
\hline Gravida-2 & - & - & $56(38.5 \%)$ & $9(25.1 \%)$ & $65(34.9 \%)$ \\
\hline Gravida-3 and Above & - & $2(40 \%)$ & $6(10.5 \%)$ & $30(28.5 \%)$ & $41(15.1 \%)$ \\
\hline Total & 3 & 6 & 142 & 35 & 186 \\
\hline
\end{tabular}

\begin{tabular}{|c|c|c|c|c|c|}
\hline \multirow[b]{2}{*}{ Weight in kg } & \multicolumn{2}{|c|}{ Microalbuminuria Positive } & \multicolumn{2}{|c|}{ Microalbuminuria Negative } & \multirow[b]{2}{*}{ Subjects } \\
\hline & $\begin{array}{c}\text { Normotensive } \\
\text { (Weeks) }\end{array}$ & $\begin{array}{l}\text { Hypertensive } \\
\text { (Weeks) }\end{array}$ & $\begin{array}{c}\text { Normotensive } \\
\text { (Weeks) }\end{array}$ & $\begin{array}{c}\text { Hypertensive } \\
\text { (Weeks) }\end{array}$ & \\
\hline $45-49$ & $1(50 \%)$ & $1(20 \%)$ & $15(10.5 \%)$ & $2(5.7 \%)$ & $19(10.7 \%)$ \\
\hline $50-54$ & $2(50 \%)$ & $5(80 \%)$ & $102(71.8 \%)$ & $26(74.3 \%)$ & $135(72.1 \%)$ \\
\hline$>55$ & - & - & $25(17.7 \%)$ & $7(20 \%)$ & $32(17.2 \%)$ \\
\hline Total & 3 & 6 & 142 & 35 & 186 \\
\hline
\end{tabular}

\begin{tabular}{|c|c|c|c|c|c|}
\hline \multirow{2}{*}{$\begin{array}{l}\text { Platelet Count in Lakhs } \\
\text { cu. } \mathbf{~ m m}\end{array}$} & \multicolumn{2}{|c|}{ Microalbuminuria Positive } & \multicolumn{2}{|c|}{ Microalbuminuria Negative } & \multirow[b]{2}{*}{ Subjects } \\
\hline & $\begin{array}{c}\text { Normotensive } \\
\text { (Weeks) }\end{array}$ & $\begin{array}{l}\text { Hypertensive } \\
\text { (Weeks) }\end{array}$ & $\begin{array}{c}\text { Normotensive } \\
\text { (Weeks) }\end{array}$ & $\begin{array}{l}\text { Hypertensive } \\
\text { (Weeks) }\end{array}$ & \\
\hline$<1.5$ & $3(50 \%)$ & $4(80 \%)$ & $10(7.1 \%)$ & $5(14.2 \%)$ & $22(9.2 \%)$ \\
\hline $1.5-1.9$ & - & $1(20 \%)$ & $130(91.5 \%)$ & $28(80 \%)$ & $159(88.5 \%)$ \\
\hline$>2$ & - & $1(20 \%)$ & $2(1.4 \%)$ & $2(5.8 \%)$ & $5(2.3 \%)$ \\
\hline Total & 3 & 6 & 142 & 35 & 186 \\
\hline
\end{tabular}

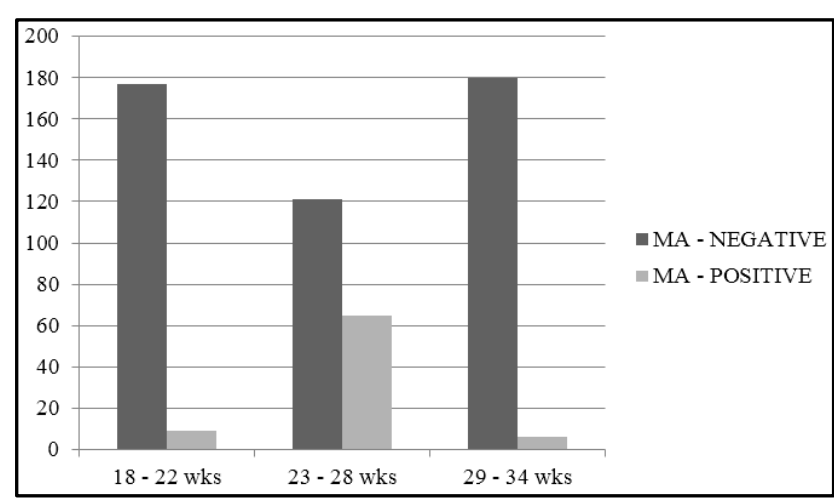

Observations in Microalbuminuria Tested Subjects at Various Weeks of Gestation

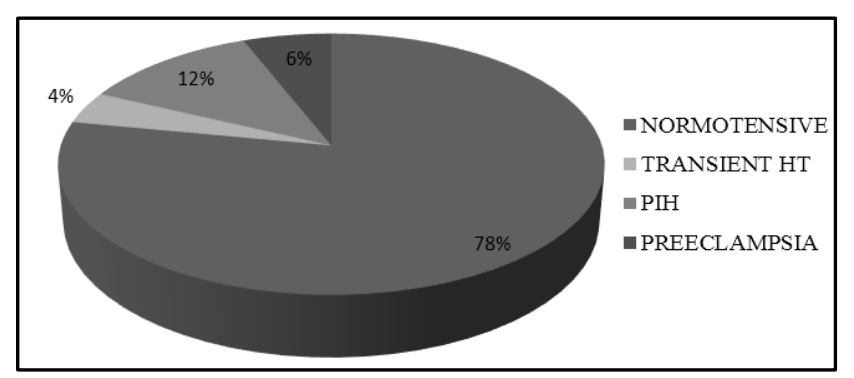

Pattern of HT Disorders in Microalbuminuria Tested Patients

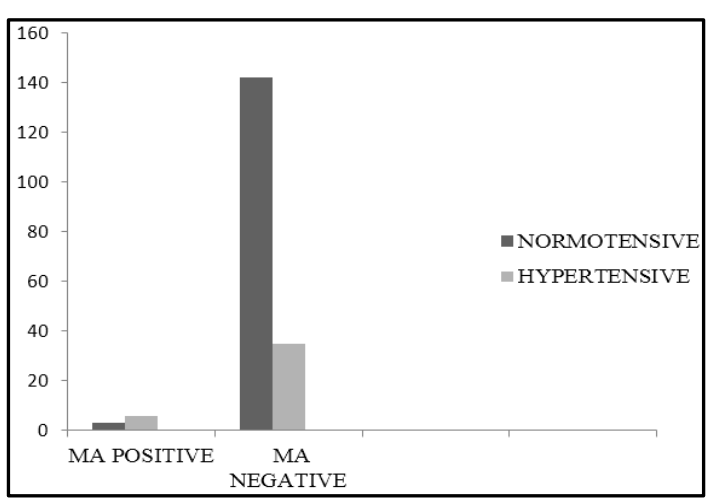

Observations in Microalbuminuria Tested Patients at 18-22 Weeks of Gestation

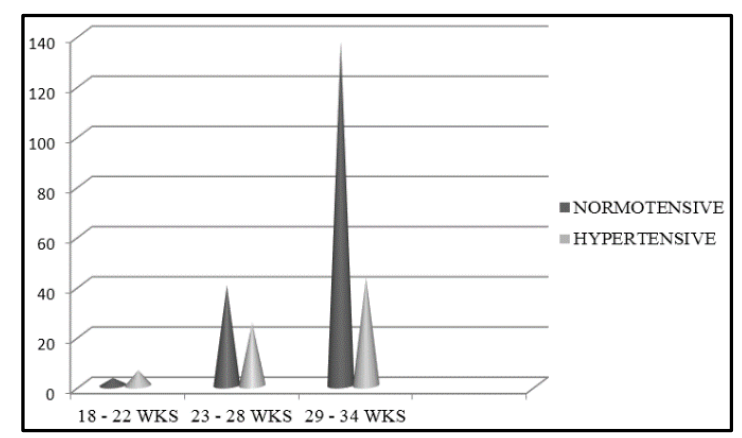

Normotensive and Hypertensive Disorders in Microalbuminuria Positive Subjects 


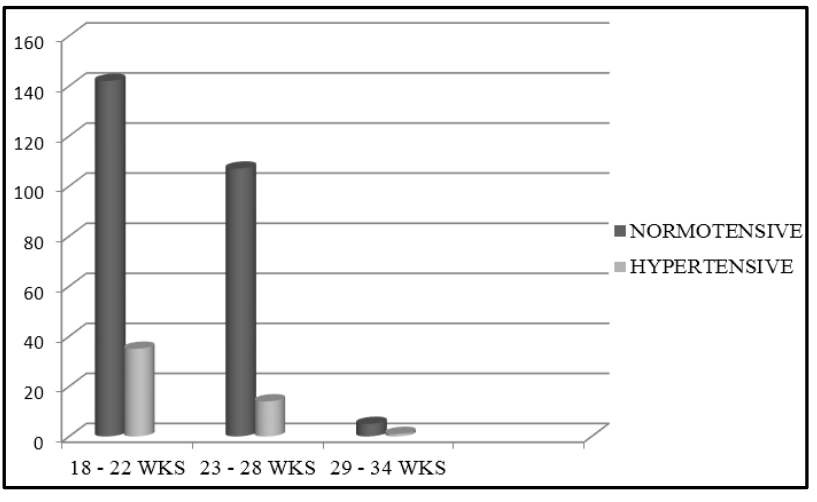

Normotensive and Hypertensive Disorders in Microalbuminuria Negative Subjects

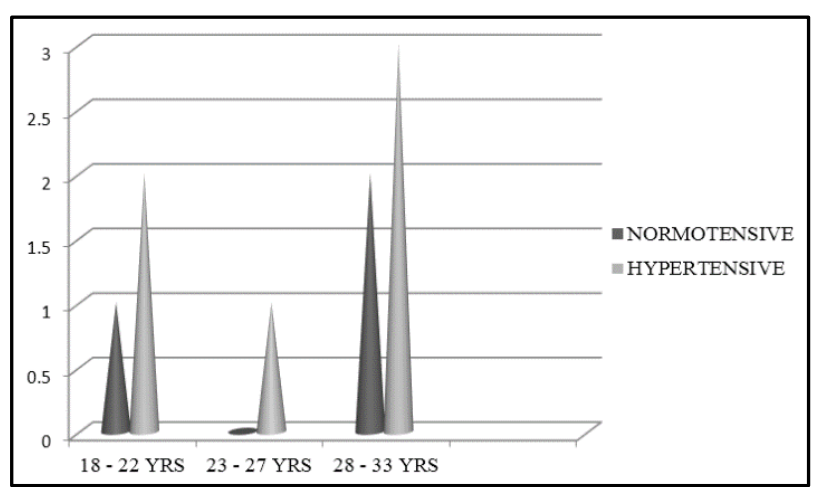

Normotensive and Hypertensive Disorders in Relation to Maternal Age in Microalbuminuria Positive Subjects at 1822 Weeks

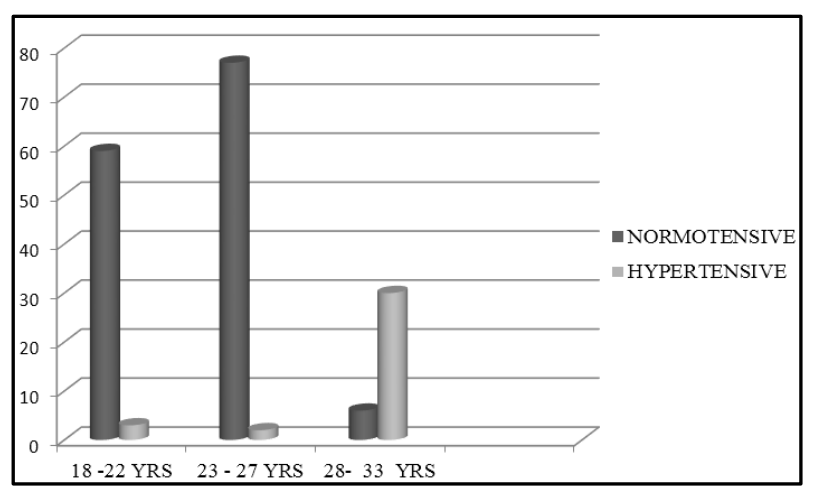

Normotensive and Hypertensive Disorders in Relation to Maternal Age in Microalbuminuria Negative Subjects at 18-22 Weeks

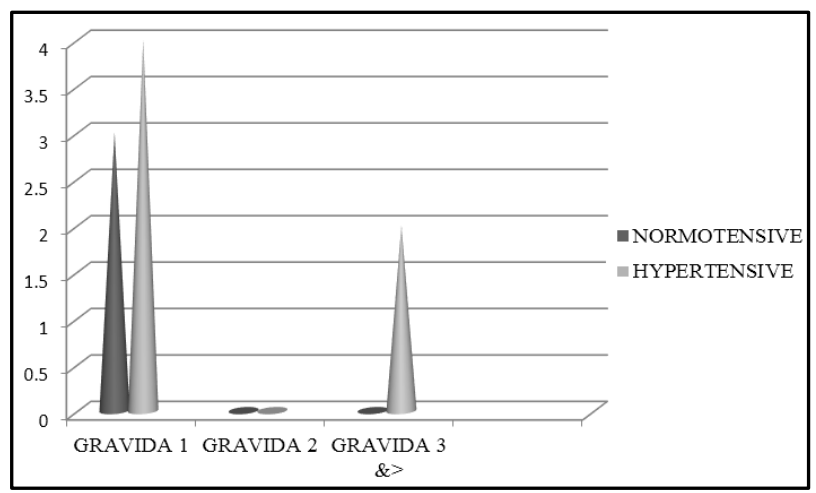

Normotensive and Hypertensive Disorders in Relation to Gravidity in Microalbuminuria Positive Subjects at 18-22 Weeks

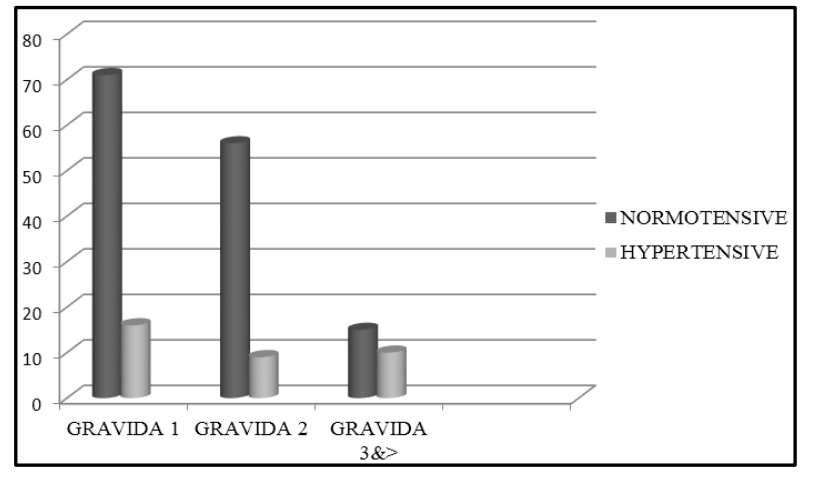

Normotensive and Hypertensive Disorders in Relation to Gravidity in Microalbuminuria Negative Subjects at 18-22 Weeks

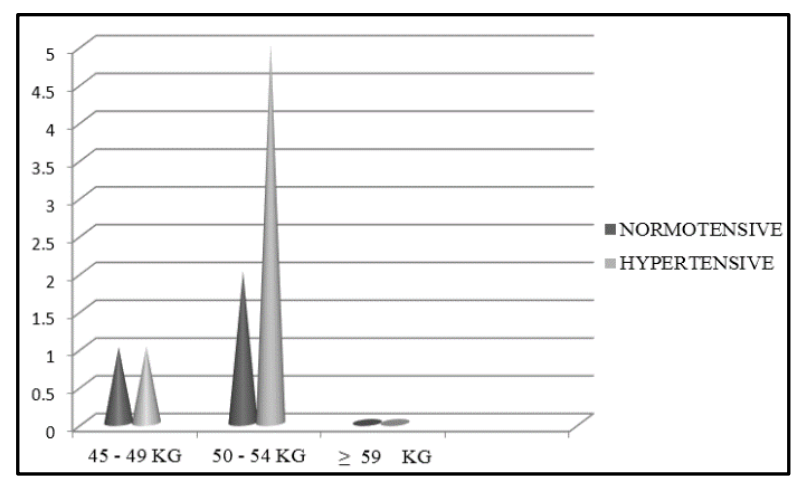

Normotensive and Hypertensive Disorders in Relation to Weight in Microalbuminuria Positive Subjects at 18-22 Weeks

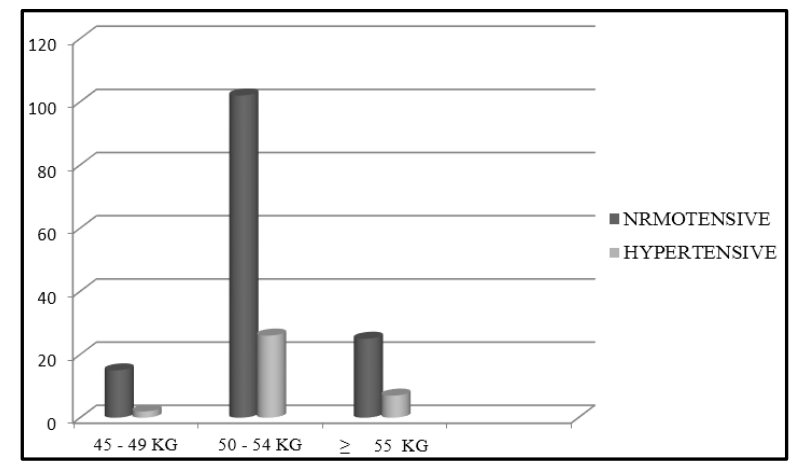

Normotensive and Hypertensive Disorders in Relation to Weight in Microalbuminuria Negative Subjects at 18-22 Weeks

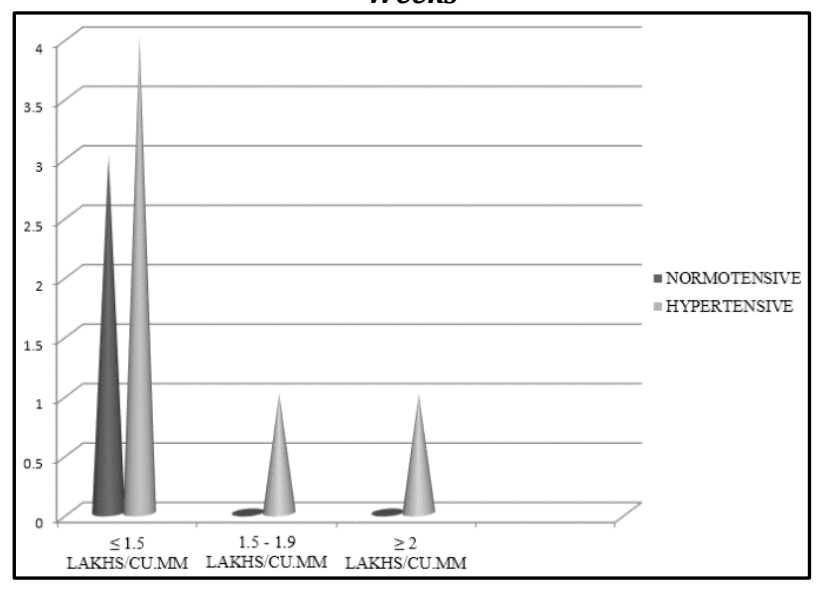

Normotensive and Hypertensive Disorders in Relation to Platelet Count in Microalbuminuria Positive Subjects at 18-22 Weeks 


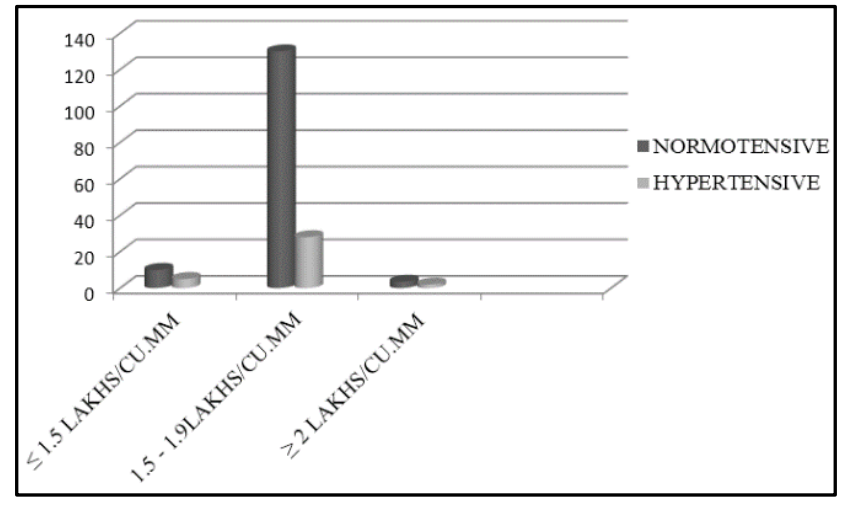

Normotensive and Hypertensive Disorders in Relation to Platelet Count in Microalbuminuria Negative Subjects at 18-22 Weeks

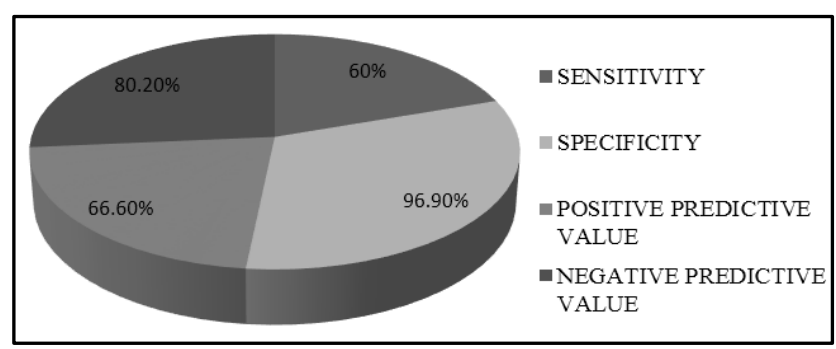

Statistical Analysis in Our Study

\section{DISCUSSION}

With the recent development of indirect latex slide test for detection of urinary albumin, it is now possible to detect microalbuminuria that would have got unnoticed in the past. Microalbuminuria is defined as urinary excretion of albumin that is persistently increased above normal (30-300 mg/litre), but below the sensitivity of conventional semi-quantitative test. It's presence indicates glomerular dysfunction resulting from generalised microvascular damage.

Lopez-Espinoza et al (1986)-In their study by radioimmunoassay of 24 hours urine collection found that rates of albumin excretion increase during pregnancy between 25-36 weeks of gestation, although remained within nonpregnant range and could find no evidence that gross proteinuria is preceded by gradual increase in microalbuminuria.

Irgine et al (1986)-studied 54 subjects at 24-41 weeks by immunoelectrophoretic methods and concluded that predictive value of urinary albumin concentration for preeclampsia had sensitivity of $61 \%$, specificity $87 \%, 61 \%$ positive, and $87 \%$ negative predictive value.

Rodriguez et al (1988)-analysed samples at 20 and 24 weeks by double antibody radioimmunoassay, the albumin concentration $>11 \mu \mathrm{g} / \mathrm{mL}$ was taken as predictive of preeclampsia and reported sensitivity of $50 \%$, specificity $82 \%$, $26 \%$ positive, and $93 \%$ negative predictive value.

Nakamura et al (1992)-studied random urine samples for microalbumin by radioimmunoassay at 20 and 30 weeks. $8.5 \%$ of them ultimately developed PIH and rest remained normotensive. In normotensive and PIH women at 30 weeks, the fasting urinary albumin had increased to $127 \%$ and $138 \%$ of the value at 20 weeks respectively.

Das et al (1996)-studied pregnant women and found that $17 \%$ developed microalbuminuria and out of this $60 \%$ developed $\mathrm{PIH}$ while $5.8 \%$ of microalbuminuria negative patients developed PIH. Sensitivity of the test was $68.42 \%$ and specificity $91.84 \%$.

North RA et al (1996) women with past pre-eclampsia are at increased risk of developing chronic hypertension and microalbuminuria. The study raises the long-term health implications of pre-eclampsia.

Kaplan et al (1996) The study group consisted of 48 women in whom pregnancy had been complicated by preeclampsia. Urinary albumin excretion rate, blood pressure, and renal function parameters were assessed 2-4 months and 3-5 years after the pregnancy. Results were compared with those in 44 women after normal pregnancy. A history of pregnancy complicated by pre-eclampsia is associated with a high occurrence of microalbuminuria.

Salako BL et al (2003) Studied urinary microalbumin excretion in one hundred healthy normotensive Nigerian pregnant women attending the antenatal clinic and followed them till delivery. Urinary microalbumin excretion when used as a single test at booking appeared to predict pre-eclampsia with a high sensitivity, but a low positive predictive value.

Brantsma et al (2006) The hypothesis that high urinary albumin excretion indicating mild renal damage may precede development of hypertension was tested. Baseline urine albumin excretion was significantly associated with the risk for developing hypertension (Odds ratio 2.29; 95\% confidence interval 1.77 to 2.95 per 10 -fold increase of urine albumin excretion). Kattah AG et al (2013) In this study, we present evidence that women with a history of hypertension in pregnancy are at significantly increased risk for microalbuminuria later in life as compared to those with normotensive pregnancies.

\section{IN OUR STUDY AT \\ 18-22 Weeks}

1. 177 (95.04\%) women were microalbuminuria negative.

2. $9(04.96 \%)$ women were microalbuminuria positive and of these

3. $6(66 \%)$ of them developed hypertensive disorder and

4. $3(33 \%)$ remained normotensive.

\section{At 23-28 Weeks}

1. $121(65.18 \%)$ women were microalbuminuria negative.

2. $65(34.92 \%)$ women were microalbuminuria positive and of these

3. $25(38 \%)$ of them developed hypertensive disorder and

4. $40(62 \%)$ remained normotensive.

\section{By 29-34 Weeks}

1. $6(3.24 \%)$ women were microalbuminuria negative.

2. $180(96.76 \%)$ women were microalbuminuria positive and of these.

3. $43(23.75 \%)$ developed hypertensive disorder and

4. $137(76.25 \%)$ remained normotensive.

\section{STATISTICAL ANALYSIS}

\section{At 18-22 Weeks}

- Sensitivity of the test is $60 \%$.

- $\quad$ Specificity of the test is $96.9 \%$.

- Positive predictive value is $66.6 \%$.

- Negative predictive value is $80.2 \%$. 


\section{At 23-28 Weeks}

- Sensitivity of the test is $63.16 \%$.

- $\quad$ Specificity of the test is $72.27 \%$.

- Positive predictive value is $40 \%$.

- $\quad$ Negative predictive value is $87.27 \%$.

\section{At 29-34 weeks}

- Sensitivity of the test is $93.37 \%$.

- $\quad$ Specificity of the test is $3.03 \%$.

- Positive predictive value is $22.42 \%$.

- Negative predictive value is $80 \%$.

Application of Kolmogorov-Smirnov Test signified that microalbumin excretion was statistically significant in those who developed hypertension. Occurrence of hypertensive disorder in primigravida was highly significant $\mathrm{P}<0.001$ (99\% confidence interval of the mean variable). Occurrence of hypertensive disorder in women with platelet count $<1.5$ was significantly seen at $18-22$ weeks. $\mathrm{P}>0.001$.

\section{CONCLUSION}

- Microalbuminuria seems to be a clinical test, which can be used for prediction of PIH, Pre-eclampsia around 18-22 weeks, later on it is not useful.

- Women at the extremes of age (18-22 yrs. and 28-33 yrs.) significantly correlated with microalbuminuria and hypertensive disorders at 18-22 weeks.

- Primigravidae who are microalbuminuria positive at 1822 weeks are much more predisposed for hypertensive disorders.

- Maternal weight was found to be an independent variable with microalbuminuria for predicting hypertensive disorders.

- Women with platelet count less than 1.5 lakhs/cubic mm found to be correlated with microalbuminuria at 18-22 weeks.

- There was no correlation between socioeconomic status, past gestational complications, height, haemoglobin, or blood group with microalbuminuria positivity.

\section{REFERENCES}

1. Lopez-Espinoza I, Dhar H, Humphreys S, et al. Urinary albumin excretion in pregnancy. Br J Obstet Gynaecol 1986;93(2):176-81.

2. Irgine ML, Hemmingsen L, Holf, et al. Immunoelectrophoretic analysis of urinary albumin. J Clini Chem Acta 1986;157:295.

3. Rodriguez MH, Masaki DI, Mestman J, et al. Calcium/creatinine ratio and microalbuminuria in the prediction of pre-eclampsia. Am J Obstet Gynaecol 1988;159(6):1452-5.

4. Nakamura T, Ito M, Oshimura T, et al. Usefulness of the urinary microalbumin/creatinine ratio in predicting pregnancy-induced hypertension. Int J Gynaecol Obstet 1992;37(2):99-103.

5. Das V, Bhargava T, Das SK, et al. Microalbuminuria: a predictor of pregnancy-induced hypertension. Br J Obstet Gynaecol 1996;103(9):928-30.

6. North RA, Simmons D, Barnfather D, et al. What happens to women with pre-eclampsia? Microalbuminuria and hypertension following pre-eclampsia. Aust N Z J Obstet Gynaecol 1996;36(3):233-8.

7. Bar J, Kaplan B, Wittenberg C, et al. Microalbuminuria after pregnancy complicated by pre-eclampsia. Nephrol Dial Transplant 1999;14(5):1129-32.

8. Salako BL, Olayemi O, Odukogbe AT, et al. Microalbuminuria in pregnancy as a predictor of preeclampsia and eclampsia. West Afr J Med 2003;22(4): 295-300.

9. Brantsma $\mathrm{AH}$, Bakker SJ, de Zeeuw D, et al. Urinary albumin excretion as a predictor of the development of hypertension in the general population. J Am Soc Nephrol 2006;17(2):331-5.

10. Kattah AG, Asad R, Scantlebury DC, et al. Hypertension in pregnancy is a risk factor for microalbuminuria later in life. J Clin Hypertens (Greenwich) 2013;15(9):617-23. 